\title{
Atrial Pacing During a Narrow QRS Complex Tachycardia: What is the Mechanism?
}

\author{
FRED MORADY, M.D.
}

From the Division of Cardiology, Department of Internal Medicine, University of Michigan Medical Center, Ann Arbor, Michigan

\section{Case Presentation}

A 25 -year-old woman with a 5-year history of paroxysmal supraventricular tachycardia underwent an electrophysiologic procedure. The baseline sinus cycle length, atrial-His-bundle (AH) interval, and His-bundle-ventricular (HV) interval were 750, 60, and $50 \mathrm{msec}$, respectively. Paroxysmal supraventricular tachycardia with a cycle length of $320 \mathrm{msec}$ was induced by programmed atrial stimulation with a single extrastimulus (Fig. 1). The response to atrial pacing at a cycle length of 260 msec during the tachycardia is shown in Figure 2 . The tachycardia was terminated by atrial pacing at a cycle length of $240 \mathrm{msec}$. Upon termination of the tachycardia, atrial pacing at a cycle length of 260 msec was performed during sinus rhythm (Fig. 3). Ventricular overdrive pacing always terminated the tachycardia. During ventricular pacing, the atrial activation sequence was the same as during tachycardia. What was the effect of

This manuscript was processed by a guest editor.

J Cardiovasc Electrophysiol, Vol. 13, pp. 296-298, March 2002.

Address for correspondence: Fred Morady, M.D., Division of Cardiology, Department of Internal Medicine, University of Michigan Medical Center, 1500 E. Medical Center Drive, B1F245, Ann Arbor, MI 48109-0022. Fax: 734-936-7026; E-mail: fmorady@umich.edu atrial pacing on the tachycardia, and what is the mechanism of the tachycardia?

\section{Commentary}

There are four possible responses to atrial overdrive pacing at a cycle length shorter than the tachycardia cycle length during paroxysmal supraventricular tachycardia: entrainment; termination; termination and reinduction; or dissociation of the atrial electrograms from the tachycardia. Figure 4 demonstrates that atrial pacing at a cycle length of $260 \mathrm{msec}$ gradually accelerated the tachycardia from its baseline cycle length of $320 \mathrm{msec}$ to a cycle length of 270 msec. Upon cessation of pacing, the tachycardia cycle length returned to $320 \mathrm{msec}$ within two cycles. This response is consistent with either entrainment or termination and reinduction of the tachycardia.

The classic criteria for entrainment of a tachycardia include acceleration of the tachycardia to the pacing cycle length and resumption of the original tachycardia upon cessation of pacing. In this case, the tachycardia cycle length shortened during pacing, but never reached the pacing cycle length of $260 \mathrm{msec}$. This can be explained by decremental conduction in the AV node during the rapid atrial pacing and does not distinguish entrainment from termination and reinduction of the tachycardia.

Note that upon cessation of pacing, the first two cycle
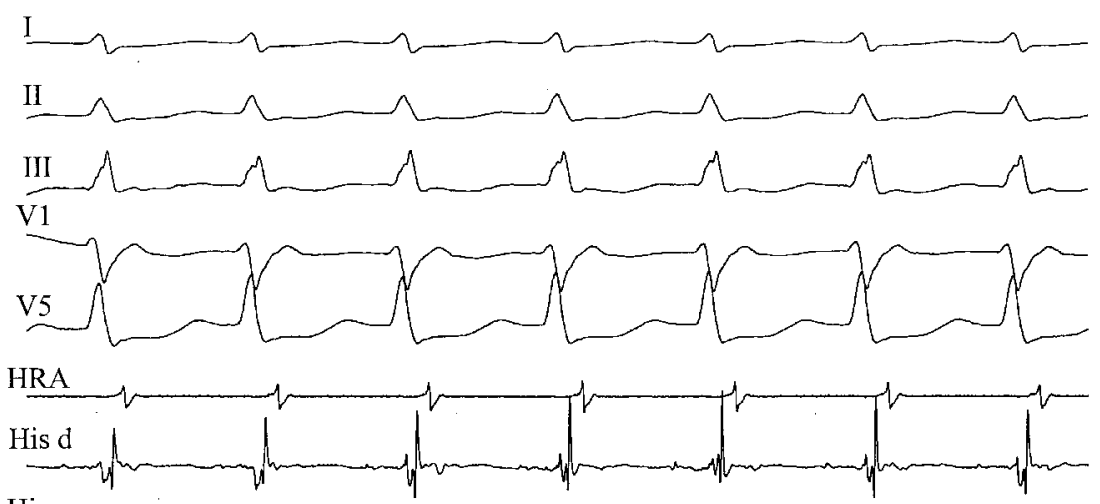

His $m$

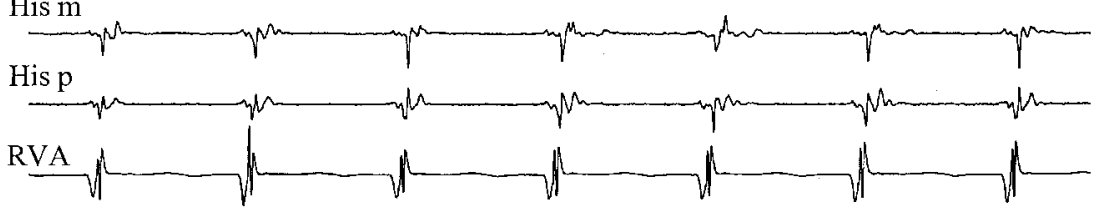

S

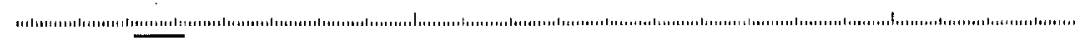
$\overline{100 \mathrm{~ms}}$
Figure 1. Narrow QRS complex tachycardia with a cycle length 320 msec. Shown are leads I, II, III, $V_{1}$, and $V_{5}$, high right atrial electrogram (HRA), distal, medial, and proximal His-bundle electrograms (His $d$, His $m$, His $p$ ), right ventricular apex electrogram (RVA), and the stimulus channel $(S)$. 
Figure 2. The last four stimuli of a train of atrial pacing at a cycle length of $260 \mathrm{msec}$ during the tachycardia. Abbreviations as in Figure 1.

Figure 3. Atrial pacing at a cycle length of $260 \mathrm{msec}$ in the setting of sinus rhythm. Abbreviations as in Figure 1.

Figure 4. Same recordings as in Figure 2, with the measurements of $R-R$ cycle lengths shown in milliseconds. The asterisk marks the last QRS complex affected by the pacing train. Abbreviations as in Figure 1 .

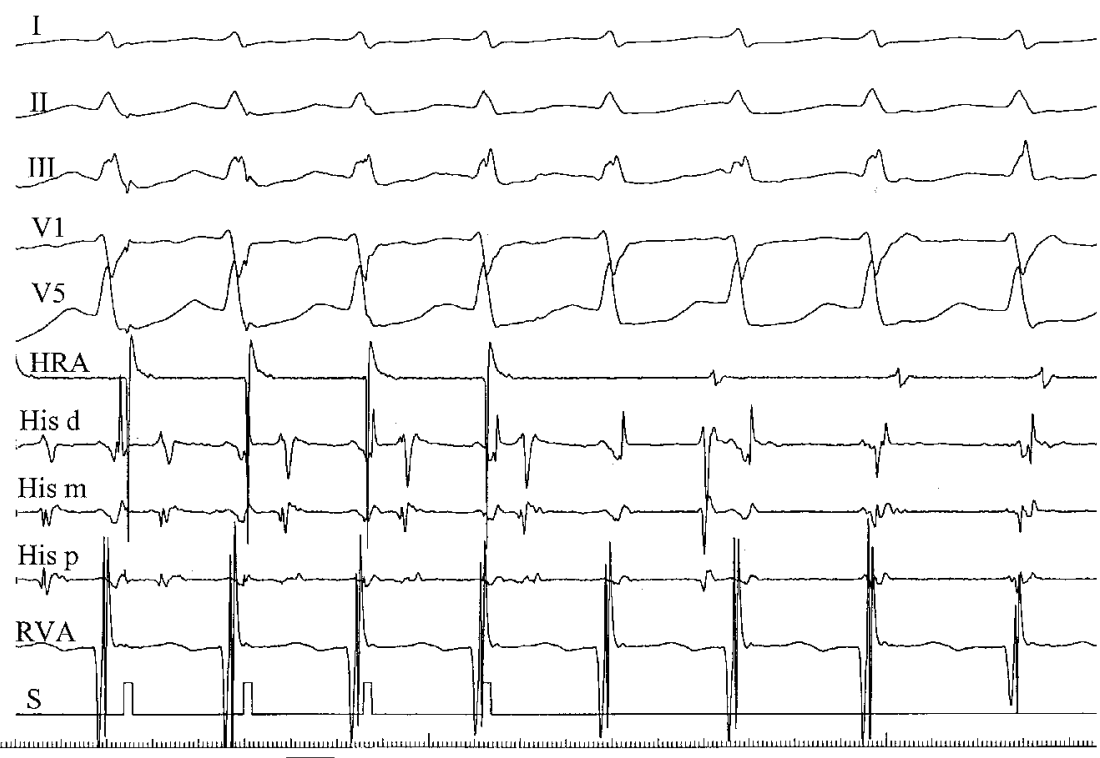

$1 \overline{00 \mathrm{~ms}}$

I

II

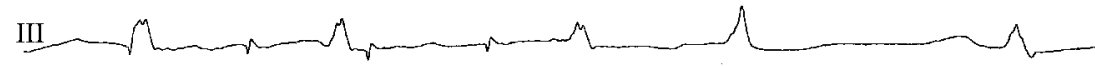
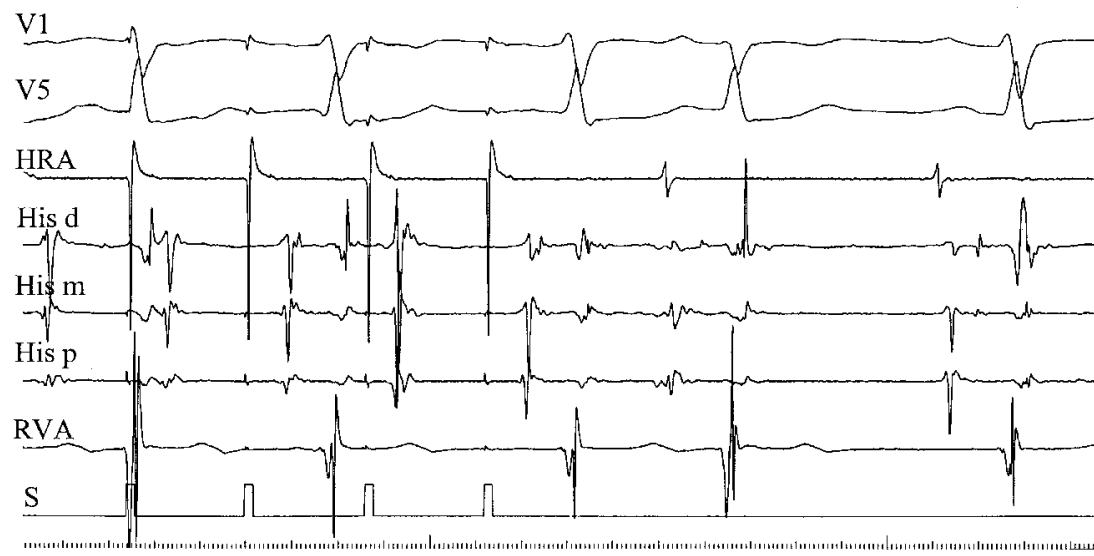

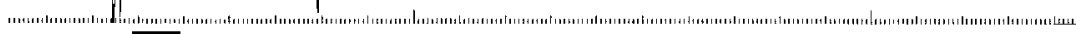
$\overline{100 \mathrm{~ms}}$

I

II

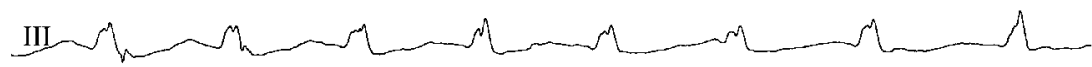
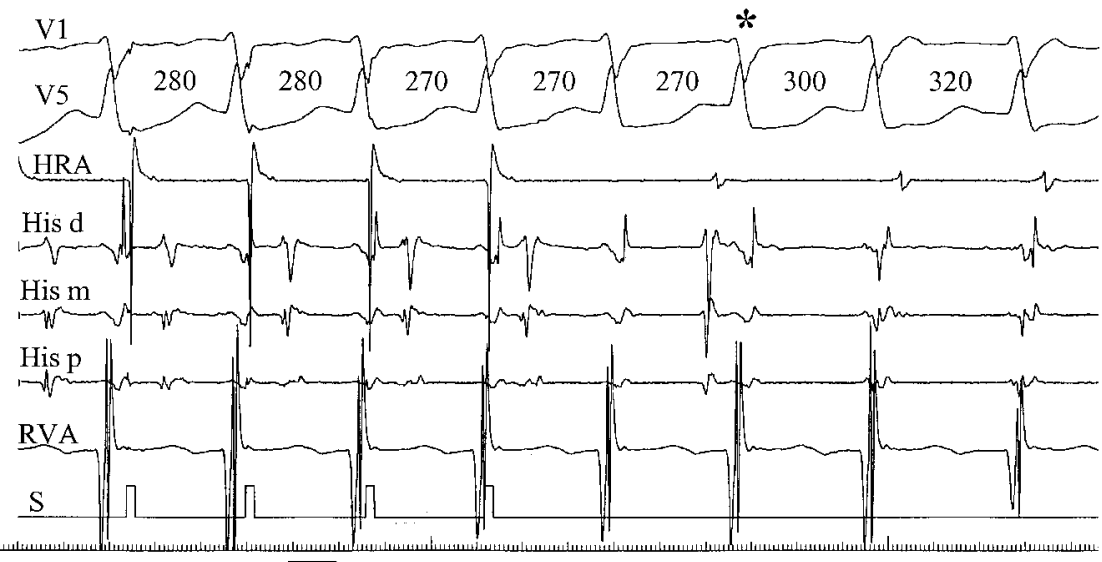
lengths both are $270 \mathrm{msec}$, which is close to the pacing cycle length of $260 \mathrm{msec}$ (Fig. 4). This indicates that the last QRS complex affected by the atrial pacing is the second one that follows the cessation of pacing, which is consistent with conduction through the slow pathway of an AV nodal reentrant circuit (Fig. 4). The alternative explanation of termination and reinduction of the tachycardia also would require conduction through a slow AV nodal pathway, because the AV interval that follows the first atrial electrogram upon cessation of pacing is too short to be consistent with conduction from the atrium to the ventricle.

Although the pacing cycle length was exactly the same, the response to atrial pacing in the setting of sinus rhythm was very different than the response during tachycardia. As seen in Figure 3, atrial pacing during sinus rhythm resulted in 2:1 AV block, with short AV intervals in the conducted complexes. This response occurred within a few seconds after termination of the tachycardia and, therefore, is unlikely to have been attributable simply to fluctuations in autonomic tone. The most likely explanation is that atrial pacing during sinus rhythm engaged a fast AV nodal pathway that had a longer anterograde block cycle length than the slow AV nodal pathway that was engaged by pacing during the tachycardia.

The observations in this case are best explained by the presence of dual $\mathrm{AV}$ nodal pathways and $\mathrm{AV}$ nodal reentrant tachycardia. Although orthodromic AV reentry may coexist with dual AV nodal pathways, the septal atrial electrogram in this case occurs before the end of the QRS complex, and the VA interval of $45 \mathrm{msec}$ is too short for $\mathrm{AV}$ reentry.

Although none of the findings discussed thus far absolutely rule out an atrial tachycardia, they do make this possibility unlikely. An atrial tachycardia would require the highly improbable scenario of dual AV nodal pathways, conduction of the atrial tachycardia to the ventricles through only the slow pathway, conduction of atrial paced complexes through only the slow pathway only when pacing is performed during tachycardia (not during sinus rhythm), and origination of the tachycardia very close to the AV node. A ventricular-atrial-ventricular ("VAV") response upon cessation of ventricular pacing that entrained the tachycardia might have provided definitive evidence against an atrial tachycardia, but this evidence was not available because ventricular pacing always terminated the tachycardia.

There is one other noteworthy observation in Figure 1: the VA relationship is transiently altered upon the cessation of pacing. In AV nodal reentrant tachycardia, the VA relationship usually remains constant after a perturbation such as rapid atrial pacing. However, alteration of the AV relationship may occur in AV nodal reentrant tachycardia and, in this case, is explained by either rate-related delay in anterograde conduction through a lower common pathway or acceleration of retrograde conduction through the fast pathway due to rate-related delay in the anterograde slow pathway.

The tachycardia was eliminated successfully by slow pathway ablation in the posteroseptal right atrium, near the ostium of the coronary sinus. 